\title{
Does systemic inflammation trigger local exercise-induced oxidative stress in COPD?
}

\author{
C. Koechlin*, A. Couillard*, J.P. Cristol\#, P. Chanez", M. Hayot*, D. Le Gallais ${ }^{+}$, C. Préfaut*
}

Does systemic inflammation trigger local exercise-induced oxidative stress in COPD? C. Koechlin, A. Couillard, J.P. Cristol, P. Chanez, M. Hayot, D. Le Gallais, C. Préfaut. (C) ERS Journals Ltd 2004.

ABSTRACT: Inflammatory abnormalities may be involved in the inadequate basal oxidant/antioxidant balance and local exercise-induced oxidative stress in chronic obstructive pulmonary disease (COPD) patients.

The time course of oxidative stress and inflammation was investigated in 10 COPD patients and seven healthy subjects before and after local dynamic quadriceps endurance exercise at $40 \%$ of maximal strength. Venous samples were collected before, immediately after and up to $48 \mathrm{~h}$ after exercise.

At rest, levels of an oxidant released by stimulated phagocytes, the superoxide anion, were significantly higher in patients, as were plasma levels of $C$-reactive protein, tumour necrosis factor- $\alpha$ and interleukin-6, inflammatory markers. An inverse relationship was found between baseline $\mathrm{C}$-reactive protein levels and endurance time in patients. Six hours after exercise, superoxide anion release and levels of protein oxidation products, an index of oxidative stress, increased similarly in both groups, whereas thiobarbituric acid reactive substance levels, another index of oxidative stress, increased significantly only in patients. Plasma nonenzymatic antioxidant and inflammatory cytokine levels were unchanged by the exercise protocol.

The increased baseline systemic inflammation in chronic obstructive pulmonary disease patients could be related to disturbed oxidant/antioxidant balance, and, together, these may have triggered the exercise-induced oxidative stress. The absence, however, of local exercise-induced systemic inflammation suggests that additional mechanisms explain local exercise-induced oxidative stress.

Eur Respir J 2004; 23: 538-544.
*Unité Propre de Recherche et d'Enseignement Supérieur Equipe d'Accueil 701, Laboratoire de Physiologie des Interactions, Service Central de Physiologie Clinique, Hospital Arnaud de Villeneuve, "Laboratoire de Biochimie des Lipides et du Stress Oxydant, Hospital Lapeyronie, "Service de Pneumologie, Hospital Arnaud de Villeneuve, and ${ }^{+}$Laboratoire Sport Performance Santé, Montpellier, France.

Correspondence: C. Koechlin, Laboratoire de Physiologie des Interactions, Service Central de Physiologie Clinique, Hôpital Arnaud de Villeneuve, 34295 Montpellier Cedex 5, France.

Fax: 33467335923

E-mail: christelle_koechlin@hotmail.com

Keywords: Cytokine

endurance

muscle

myopathy

superoxide anion

Received: June 182003

Accepted after revision: October 312003
Considerable evidence links chronic obstructive pulmonary disease (COPD) with increased oxidative stress [1-3], defined as an imbalance between pro-oxidant and antioxidant factors in favour of the former. Since oxidative stress can induce substantial muscle alterations [4], it may be implicated in the peripheral skeletal muscle dysfunction and/or impaired exercise tolerance described in COPD patients $[5,6]$. Numerous studies have shown that cycle exercise $[7,8]$, and even exercise localised only to the quadriceps, results in systemic and muscle oxidative stress in COPD [3, 9].

Studies have also reported increased levels of circulating cytokines (interleukin (IL)-6 and -8 and tumour necrosis factor- $\alpha(\mathrm{TNF}-\alpha)$ ) and acute phase reactant protein $(\mathrm{C}$ reactive protein (CRP)), both of which reflect systemic inflammation, in the peripheral circulation of stable COPD patients $[10,11]$. This suggests that COPD affects not only the lungs but also distant organs [12], as systemic inflammation may directly or indirectly have many harmful effects, for example by inducing oxidative stress [13]. Indeed, TNF- $\alpha$ reduces the levels of intracellular glutathione, an important physiological antioxidant, by a mechanism involving intracellular oxidative stress $[13,14]$. TNF- $\alpha$ and ILs can also induce expression of the inducible form of nitric oxide synthase or enhance mitochondrial generation of reactive oxygen species (ROS) [13, 15]. Finally, neutrophils, which appear to be critical cells in the pathogenesis of COPD and its inflammatory events [14], are an important source of ROS
[16]. Two studies have already suggested that inflammatory abnormalities may be involved in the inadequate oxidant/ antioxidant balance observed in COPD patients at rest [1, 12]. However, since oxidative stress enhances inflammation through activation of redox-sensitive transcription factors that regulate the gene for inflammatory mediators [2], exercise-induced oxidative stress could, inversely, induce abnormal exerciseinduced inflammation in COPD.

Substantial evidence thus led to the present hypothesis that inflammation and oxidative stress coexist in COPD and that these two processes interact. This study investigated the time course of oxidative stress and inflammatory mediator levels at rest and after local exercise in COPD patients compared with age-matched healthy subjects.

\section{Methods and materials}

\section{Subjects}

The present study included 17 male subjects. The 10 COPD patients were all exsmokers, selected on the basis of moderateto-severe COPD according to Global initiative for Chronic Obstructive Lung Disease guidelines [17]. At evaluation, all were in a stable condition with no disease exacerbation in the preceding 2 months. None were on oral corticosteroid therapy 
and two received inhaled steroids. All patients inhaled anticholinergic or $\beta_{2}$-agonists as bronchodilator therapy. In order to avoid potentially confounding effects, subjects with cardiac failure, diabetes mellitus and alcoholism were excluded. Seven healthy age-matched nonsmoking males were recruited for the control group. Their history and physical examination revealed no cardiopulmonary dysfunction and none exhibited symptoms of lung or heart disease. Subjects in both groups were excluded if they had previously undergone prolonged bed rest or presented with a history of muscle disease. None were involved in any form of exercise training. All participants were questioned on their dietary habits to ensure that none were taking antioxidants or vitamin supplements. The research protocol was approved by the hospital ethics committee. Written consent was obtained from all subjects after they had received a complete explanation of the purposes and protocol of the study.

\section{Lung function}

Subjects underwent spirometry using a whole body plethysmograph (Transmural Bodybox 2800; Sensormedics, Yorba Linda, CA, USA). Measurements included forced vital capacity (FVC) and forced expiratory volume in one second (FEV1). Tiffeneau's ratio (FEV1/FVC) was then calculated. The values obtained were compared to the theoretical values of QUANJER et al. [18].

\section{Assessment of body composition}

Body height and weight were assessed while subjects wore light clothing and no shoes. Fat-free mass (FFM) was determined by bioelectrical impedance analysis using a body composition analyser (model TBF-300; TANITA France, Neuilly-sur-Seine, France). Weight parameters were adjusted for body surface area, to give body mass index and fat-free mass index.

\section{Level of physical activity}

Physical activity was assessed using a physical activity questionnaire adapted for older retired adults [19] and recently used in COPD patients [3]. This questionnaire provides a reliable and valid method of classifying older subjects into categories of high, medium and low physical activity. Subjects with scores of $<9$ were classified in the low physical activity or "sedentary" category.

\section{Local muscle exercise}

Maximal voluntary contraction and quadriceps endurance of the dominant leg were assessed on an exercise bench (Banc de Koch; Genin Medical, Les Angles, France) according to a previously described method [3]. The same investigator supervised the endurance tests and gave standardised verbal encouragement to all subjects. The test duration (in seconds) was termed "limit time" ( $t$ lim). Dyspnoea and muscle fatigue scores were measured at rest and immediately after exercise on a $10-\mathrm{cm}$ visual analogue scale.

\section{Study design}

The subjects abstained from strenuous physical activity from 3 days before and during the study. After an overnight fast and without breakfasting, the subjects arrived at the laboratory between 08:00 and 09:00 h for spirometric testing. They then responded to the physical activity questionnaire and resting blood samples were taken. Next, strength was evaluated and the subjects were familiarised with the endurance test procedures by performing five consecutive dynamic knee extensions. They then performed the local muscle exercise at $\sim 10: 00 \mathrm{~h}$. Blood samples were taken immediately after the local exercise, and 6, 24 and $48 \mathrm{~h}$ later. ROS production was immediately determined using a $100-\mu \mathrm{L}$ aliquot of blood. Serum (after clotting at room temperature) and plasma samples were removed after centrifugation (for $10 \mathrm{~min}$ at $1,200 \mathrm{xg}$ at $4^{\circ} \mathrm{C}$ ) and stored at $-80^{\circ} \mathrm{C}$ until analysis.

\section{Markers of systemic oxidative stress}

Levels of thiobarbituric acid reactive substances (TBARs), as markers of lipid peroxidation, were determined spectrophotometrically using the method described by YAGI [20], and expressed as micromoles of TBARs formed per litre of plasma. The reproducibility, calculated as the coefficient of variation, was $4.6 \%$. Levels of protein oxidation, another marker of oxidative stress, were also determined in plasma by spectrophotometry of advanced oxidation protein products (AOPP) in a microplate reader (Model MR 500; Dynatec, Paris, France), using the semiautomated method previously described by WITKO-SARSAT et al. [21]. AOPP concentrations were expressed as micromoles of chloramine-T equivalents per litre. The reproducibility, calculated as the coefficient of variation, was $5.2 \%$.

\section{Markers of the oxidant system}

Superoxide anion release by stimulated phagocytes was measured in triplicate at rest, immediately after exercise and $6 \mathrm{~h}$ later according to a method derived from VACHIER et al. [22]. The blood phagocytes were stimulated with $1.6 \times 10^{-6} \mathrm{M}$ phorbol myristate acetate, a soluble stimulant, and the chemiluminigenic probe, lucigenin, was then used (at a final concentration of $1.5 \times 10^{-4} \mathrm{M}$ ) to study phagocyte $\mathrm{O}_{2}{ }^{--}$production. Luminescence was recorded at $37^{\circ} \mathrm{C}$ using a $125 \mathrm{I}$ LKB Wallac Luminometer (Wallac Co., Turku, Finland). The reproducibility, calculated as the coefficient of variation, was $6.5 \%$.

\section{Markers of the antioxidant system}

Total antioxidant capacity was assayed using a quantitative colorimetric technique in kit form (Kit NX2331; Randox, Mauguio, France) according to the supplier's instructions. The reproducibility, calculated as the coefficient of variation, was $<1 \%$. Plasma vitamin E levels were measured by highperformance liquid chromatography according to the method described by CACHIA et al. [23]. As blood vitamin E level strongly correlates with triglyceride and cholesterol levels, the sum of their levels was assessed using a routine enzymatic method (Boehringer Mannheim, Meyland, France). The reproducibility, calculated as the coefficient of variation, was $5.6 \%$. Serum uric acid (coefficient of variation $<2 \%$ ) and albumin (coefficient of variation $<1 \%$ ) levels were also determined by routine biochemical assay on an AU2700 Olympus analyser (Olympus, Rungis, France).

\section{Markers of systemic inflammation}

CRP levels, as nonspecific markers of inflammation, were determined by means of an immunoturbidimetric assay 
using an AU2700 Olympus analyser. Olympus latex reagents (Olympus Diagnostica, Olympus, O'Callaghan's Mills, Ireland) were used for sensitive (range $0.5-5 \mathrm{mg} \cdot \mathrm{L}^{-1}$ ) and highly sensitive $\left(0.05-2 \mathrm{mg} \cdot \mathrm{L}^{-1}\right)$ application.

Plasma concentrations of IL-6 and TNF- $\alpha$ were assayed using a quantitative high-sensitivity enzyme-linked immunosorbent assay (ELISA) in kit form (R\&D Systems, Minneapolis, MN, USA) according to the supplier's instructions. The kit's detection limits were $0.12 \mathrm{pg} \cdot \mathrm{mL}^{-1}$ for $\mathrm{TNF}-\alpha$ and $0.039 \mathrm{pg} \cdot \mathrm{mL}^{-1}$ for IL-6; the reproducibility, calculated as the coefficient of variation, was 5.6 and $7.4 \%$, respectively, according to the supplier.

\section{Statistical analysis}

Data are presented as mean \pm SEM. Between-group comparisons of basal variables were analysed using unpaired t-tests. When the normality assumption (Shapiro-Wilk test) and/or homogeneity of variances (Bartlett's statistic) were not obtained, a Mann-Whitney U-test was used. Differences in the time courses of the biological markers following local exercise between COPD patients and controls were assessed by two-way analysis of variance followed by Tukey's pairwise multiple comparison procedure. Significance was set at the 0.05 level.

\section{Results}

\section{Anthropometric and spirometric data}

Between-group differences were observed for spirometric function, with the COPD group showing moderate-to-severe airflow obstruction. Both groups were sedentary, with similar low levels of physical activity (table 1).

\section{Systemic oxidative stress and inflammation status at rest}

Resting plasma TBARs and AOPP levels were similar in the two groups (table 2).

$\mathrm{O}_{2} \cdot{ }^{-}$release by stimulated phagocytes was significantly greater in the COPD patients $(\mathrm{p}<0.05)$ (fig. 1). No significant difference in resting antioxidant levels was found between the groups (table 2). Resting plasma CRP, TNF- $\alpha$ and IL-6 levels were all significantly raised in the COPD patients (table 2).

Table 1. - Subject characteristics

\begin{tabular}{lcc}
\hline & COPD & Control \\
\hline Subjects n & 10 & 7 \\
Age yrs & $60 \pm 3$ & $58 \pm 2$ \\
Height cm & $168 \pm 3$ & $174 \pm 6$ \\
Weight kg & $74 \pm 5$ & $76 \pm 4$ \\
BMI kg $\cdot \mathrm{m}^{-2}$ & $26 \pm 1$ & $25 \pm 1$ \\
FFM kg & $53 \pm 3$ & $56 \pm 3$ \\
FFMI kg.m ${ }^{-2}$ & $18.8 \pm 0.8$ & $18.5 \pm 0.8$ \\
FEV1 L & $1.21 \pm 0.09^{* * *}$ & $3.60 \pm 0.26$ \\
FEV1 \% pred & $41 \pm 2^{* * *}$ & $107 \pm 5$ \\
FEV $1 /$ FVC & $51.0 \pm 3.4^{* * *}$ & $80.9 \pm 2.0$ \\
Physical activity score & $7.4 \pm 1.2$ & $8.7 \pm 1.4$ \\
\hline
\end{tabular}

Data are presented as mean \pm SEM. COPD: chronic obstructive pulmonary disease; BMI: body mass index; FFM: fat-free mass; FFMI: fat-free mass index; FEV1: forced expiratory volume in one second; FVC: forced vital capacity; $\%$ pred: per cent predicted. $* * *$ : $\mathrm{p}<0.001$ versus controls.
Table 2.-Oxidative stress, nonenzymatic antioxidants and inflammation status at rest

\begin{tabular}{lcc}
\hline & COPD & Control \\
\hline Subjects $\mathrm{n}$ & 10 & 7 \\
TBARs $\mathrm{nM}$ & $0.82 \pm 0.22$ & $0.78 \pm 0.02$ \\
AOPP $\mu \mathrm{M}$ chloramine-T equivalents & $17.1 \pm 1.3$ & $17.8 \pm 1.1$ \\
Total antioxidant capacity $\mathrm{mM}$ & $1.43 \pm 0.03$ & $1.46 \pm 0.03$ \\
Vitamin $\mathrm{E} \times 10^{3} /(\mathrm{TG}+\mathrm{CL})$ & $4.65 \pm 0.26$ & $4.33 \pm 0.18$ \\
Uric acid $\mu \mathrm{M}$ & $308 \pm 25.6$ & $324 \pm 19.4$ \\
Albumin $\mathrm{g} \cdot \mathrm{L}^{-1}$ & $45.1 \pm 0.85$ & $48 \pm 1.47$ \\
$\mathrm{CRP} \mathrm{mg} \cdot \mathrm{L}^{-1}$ & $9.3 \pm 2.4^{*}$ & $1.3 \pm 0.3$ \\
TNF- $\alpha \mathrm{pg} \cdot \mathrm{mL}^{-1}$ & $1.82 \pm 0.15^{* *}$ & $0.92 \pm 0.16$ \\
IL-6 $\mathrm{pg} \cdot \mathrm{mL}^{-1}$ & $9.46 \pm 2.91^{*}$ & $1.44 \pm 0.20$ \\
\hline
\end{tabular}

Data are presented as mean \pm SEM. COPD: chronic obstructive pulmonary disease; TBARs: thiobarbituric acid reactive substances; AOPP: advanced oxidation protein products; TG: triglycerides; CL: cholesterol; CRP: C-reactive protein; TNF- $\alpha$ : tumour necrosis factor- $\alpha$; IL: interleukin. ${ }^{*} * *$ : $\mathrm{p}<0.05, \mathrm{p}<0.01$ versus controls.

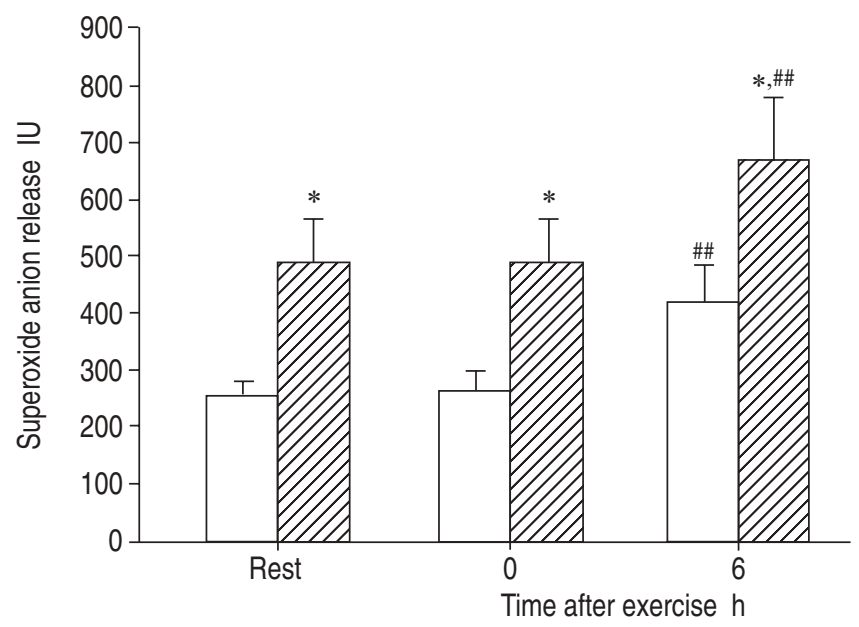

Fig. 1.- Mean superoxide anion release by stimulated phagocytes at rest, immediately after exercise and $6 \mathrm{~h}$ later in controls $(\square)$ and chronic obstructive pulmonary disease patients $(\mathbb{Z})$. Data are presented as mean \pm SEM. *: $\mathrm{p}<0.05$ versus controls; ${ }^{\#+}: \mathrm{p}<0.01$ versus rest.

\section{Local muscle endurance}

Analysis of peripheral muscle performance showed no significant differences in maximal voluntary contraction between the COPD and control groups (26 \pm 1 versus $25 \pm 1 \mathrm{~kg})$. In contrast, muscle endurance ( $t \mathrm{lim})$ was significantly nearly two-fold lower in the COPD patients than in the healthy subjects $(174 \pm 20$ versus $317 \pm 44 \mathrm{~s} ; \mathrm{p}<0.01)$. An inverse relationship was found between baseline CRP levels and $t \mathrm{lim}$ in the COPD group ( $\mathrm{p}<0.05 ; \mathrm{r}=-0.66$ ) (fig. 2), with no such association observed for the control group. Between rest and immediately after exercise, the increase in muscle fatigue score was $7.0 \pm 0.4$ in the COPD group and $6.0 \pm 0.4$ in the healthy subjects. The dyspnoea score increased by $2.3 \pm 0.7$ in the COPD group and $2.4 \pm 0.7$ in the healthy subjects.

Time course of exercise-induced oxidative stress and inflammation

Quadriceps exercise induced a significant increase in plasma TBARs levels only in the COPD patients $6 \mathrm{~h}$ after exercise 


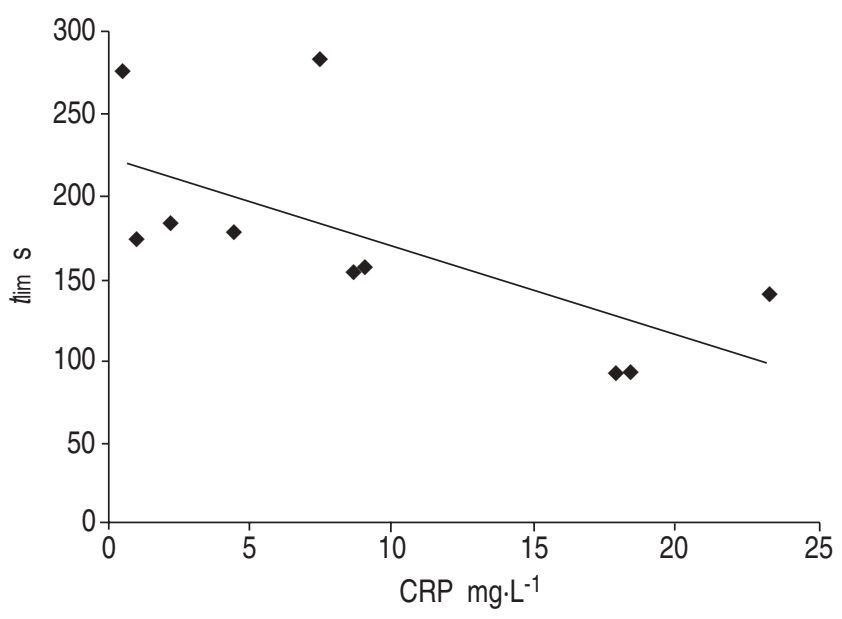

Fig. 2.-Correlation between basal blood C-reactive protein (CRP) levels and limit time $(t \mathrm{lim})$ of muscle endurance in chronic obstructive pulmonary disease patients $(\mathrm{r}=-0.66 ; \mathrm{p}<0.05)$.

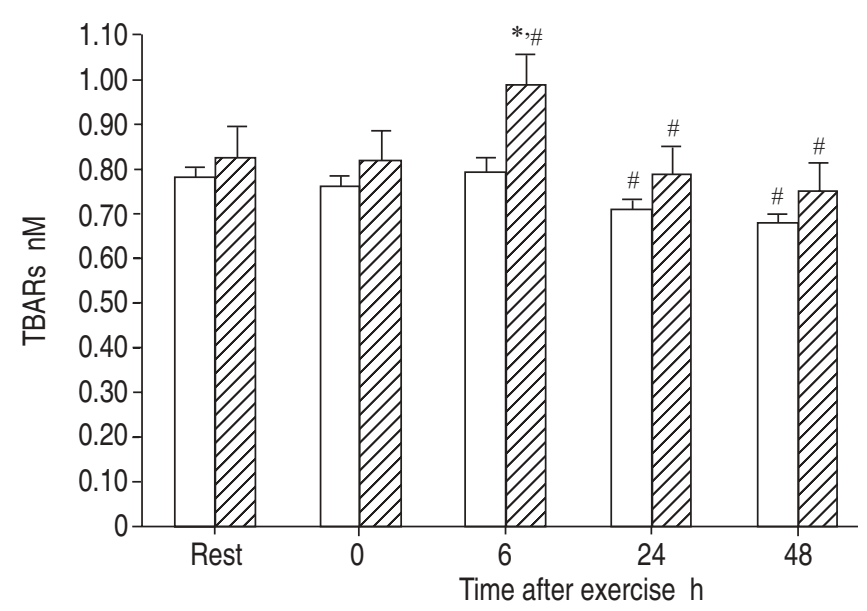

Fig. 3.-Mean levels of thiobarbituric acid reactive substances (TBARs), markers of plasma products of lipid peroxidation, at rest, immediately after exercise, and 6, 24 and 48 h later in controls ( $\square$; $\mathrm{n}=7$ ) and chronic obstructive pulmonary disease (COPD) patients ( $\mathbb{Z}$; $\mathrm{n}=10$ ). Data are presented as mean \pm SEM. Local exercise induced a significant increase in TBARs levels in COPD patients $6 \mathrm{~h}$ after exercise. TBARs levels were unchanged in the controls at this time. In contrast, after $24 \mathrm{~h}$, there was a decrease in TBARs levels compared to baseline in both groups for up to $48 \mathrm{~h} .{ }^{*}: \mathrm{p}<0.05$ versus control; ${ }^{\#}$ : $\mathrm{p}<0.05$ versus rest.

$(\mathrm{p}<0.05)$, as shown in figure 3. Afterwards, TBAR levels tended to decrease in both groups, although this decrease only became significantly different from baseline $24 \mathrm{~h}$ after exercise $(\mathrm{p}<0.05)$ (fig. 3). The plasma AOPP concentration was significantly increased $6 \mathrm{~h}$ after exercise in both groups (fig. 4), and returned towards resting levels within $48 \mathrm{~h}$. $\mathrm{O}_{2}{ }^{-}$release by stimulated phagocytes was significantly increased $6 \mathrm{~h}$ after exercise in both groups (fig. 1). Plasma nonenzymatic antioxidant and inflammatory cytokine levels were unchanged by the exercise protocol, and remained relatively constant throughout the postexercise period.

\section{Discussion}

The major findings in the COPD patients were: 1) at rest, a higher oxidant burden originating from phagocytes in parallel

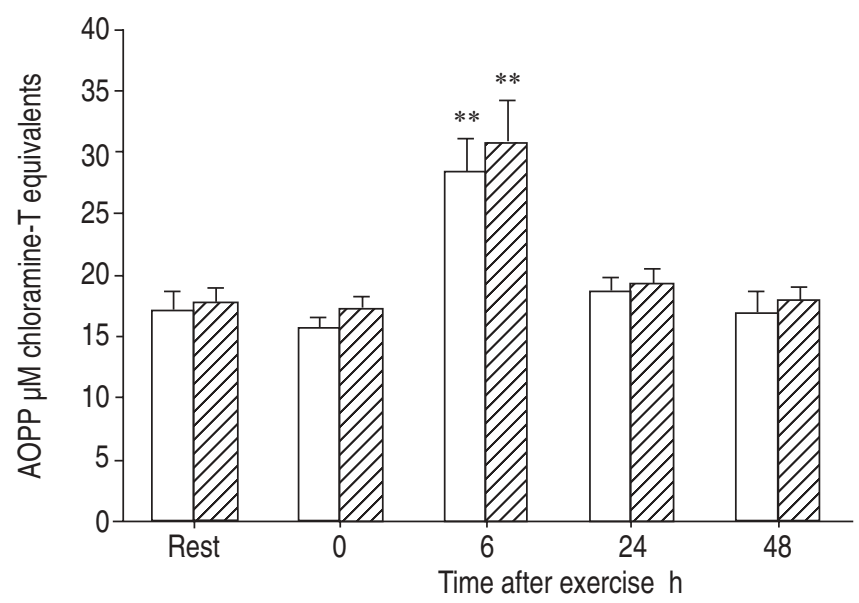

Fig. 4.-Mean advanced oxidation protein products (AOPP) levels at rest, immediately after exercise, and 6, 24 and $48 \mathrm{~h}$ later in controls $(\square ; n=7)$ and chronic obstructive pulmonary disease patients $(\mathbb{Z}$; $\mathrm{n}=10)$. Data are presented as mean \pm SEM. **: $\mathrm{p}<0.01$ versus rest.

with marked basal systemic inflammation; and 2) during exercise localised to the quadriceps, a significant increase in levels of blood markers of oxidative stress without evidence of local exercise-induced systemic inflammation.

\section{Methodology}

Quadriceps endurance. The choice of local exercise for studying muscle endurance offered three advantages. First, it minimised ventilatory response and was specific to the quadriceps [24]. Secondly, in an experiment consisting of repetitive tests using the same technique, $t \mathrm{lim}$ was found to be highly reproducible [25]. Finally, it induced systemic oxidative stress in the COPD patients [3].

Markers of oxidative stress. A global method was used for assessing oxidant-mediated lipid damage: plasma lipid peroxidation by measurement of TBARs levels. This is a nonspecific oxidative stress index that should be used with caution [26]. However, the fluorimetric method used in the present study was sufficiently sensitive and reproducible to provide a valid estimate of oxidative stress [20], as previously discussed [3]. As no one method best assesses oxidative stress, it has been recommended that many methodologies be used [27]. The detection of oxidised amino acids in biological systems also provides a fingerprint of oxidative damage and strong evidence of oxidative stress [27]. In order to assess oxidant-mediated protein damage, it was decided to measure dityrosine-containing protein cross-linking products as reflected by plasma AOPP levels [21]. Numerous recent studies have used AOPP as a reliable marker of oxidantmediated protein damage following oxidative stress [28, 29].

Markers of inflammation. Contradictory results have been reported regarding the presence of inflammatory cytokines such as TNF- $\alpha$ in the plasma of COPD patients $[10,11]$. As plasma concentrations of pro-inflammatory cytokines are very low in healthy subjects, they can only be detected using very sensitive immunoassays. Using a high-sensitivity ELISA, it was possible to quantify plasma TNF- $\alpha$ concentrations in both groups and thus to detect differences [30]. 


\section{Disturbance of oxidant status in COPD patients at rest and marked basal inflammation}

No difference in plasma TBARs or AOPP levels was found at rest between the two groups. In contrast, $\mathrm{O}_{2} \cdot{ }^{-}$release by phagocytes stimulated in vitro was significantly greater at rest in the COPD patients than in the controls, suggesting a continued oxidant burden originating from phagocytes in these patients [31]. No deficiency in plasma nonenzymatic antioxidants, as reflected by total antioxidant capacity or levels of uric acid or albumin, was noted in the COPD patients compared with the healthy age-matched subjects. Lower vitamin E levels at rest were not found in the COPD patients, which was in disagreement with the results of a previous study [3]. However, the COPD patients of the present study showed longer $t \mathrm{lim}$ than the previous patients $(174 \pm 20$ versus $136 \pm 16 \mathrm{~s})$ and lower baseline levels of TBARs and $\mathrm{O}_{2}{ }^{-}$release by phagocytes stimulated in vitro. The discrepancy between results may thus be attributable to a difference in disease severity expressed as impaired exercise tolerance and altered oxidant/antioxidant balance, with the patients of the present study less severely affected than those of the previous study [3].

Significantly increased levels of the acute phase proteins, IL- 6 and TNF- $\alpha$, were found at rest in the plasma of the stable COPD patients compared to the healthy age-matched subjects, suggesting marked chronic systemic inflammation in the patients. The increase in both basal inflammatory mediator levels and $\mathrm{O}_{2} \cdot{ }^{-}$release by phagocytes in the COPD patients suggests that inflammation and oxidative stress coexist and thus may interact in COPD. Indeed, neutrophils, which appear to be critical cells in the pathogenesis of COPD [14], and pro-inflammatory cytokines are known to constitute a main source of systemic ROS production [12, 14]. Elevated levels of cytokines, such as TNF- $\alpha$, may thus have contributed to the disturbance of the systemic basal oxidant status of the COPD patients, as reflected by increased $\mathrm{O}_{2}{ }^{-}$ release. Elevated plasma TNF- $\alpha$ levels may even promote muscle ROS production [13]. Another possibility, however, is that the baseline inflammation was a consequence of increased ROS formation through activation of the redoxsensitive transcription factors that regulate the genes for inflammatory mediators [2].

\section{Quadriceps endurance and physical activity}

The skeletal muscle dysfunction in COPD is not fully understood. The present study confirmed that COPD patients show diminished muscle endurance after local exercise despite a physical activity level similar to that of healthy subjects. This result clearly indicates that phenomena in addition to chronic inactivity are involved [9, 12]. The significantly inverse relationship between baseline CRP levels and tim found in the COPD group suggests that resting systemic inflammatory status may contribute to the impaired tolerance exercise observed in COPD.

\section{Exercise-induced oxidative stress}

The significant $(22 \%)$ increase in plasma products of lipid peroxidation in the COPD group $6 \mathrm{~h}$ after local quadriceps exercise indicates exercise-induced oxidative stress in these patients. After this point, the time course of plasma TBARs level was the same for both groups, with a significant decrease from $24 \mathrm{~h}$ onward. This decrease could be due to a delayed increase in excretion of free TBARs or TBARs adducts in urine in response to exercise [32]. Indeed, the presence of TBARs in plasma is influenced by their rate of production and excretion. TBARs are produced from polyunsaturated fatty acids, mostly membrane-associated, and their production during an exercise protocol, as described in the present study, is likely to take place in the exercised muscle. This was indicated by the lack of exercise effect on plasma antioxidant levels. TBARs are then actively excreted in the urine, which was suggested by the significant decrease in plasma TBARs levels observed from $24 \mathrm{~h}$ onward.

The significant increase in plasma AOPP level observed in both groups $6 \mathrm{~h}$ after exercise may have been due to an increase in phagocyte number in response to exercise, since WITKO-SARSAT et al. [21] showed, in uraemic patients, that AOPP level was closely related to the plasma concentration of neopterin, a marker of monocyte activation. This last result suggests that, under conditions which modulate phagocyte activation, AOPP level as a marker of oxidant-mediated protein damage should be interpreted cautiously.

The increased $\mathrm{O}_{2}{ }^{-}$release by stimulated phagocytes observed in both groups $6 \mathrm{~h}$ after local exercise suggested an increase in ROS formation in response to exercise. This increase in $\mathrm{O}_{2}{ }^{-}$release, associated with already high basal $\mathrm{O}_{2} .^{-}$release, in the COPD patients may have triggered the exercise-induced oxidative stress in this group. No change in antioxidant levels was observed after exercise in either group, however, suggesting that these nonenzymatic plasma antioxidants were probably not involved. Nevertheless, disturbances in the muscle antioxidant glutathione system observed in COPD $[5,33]$ suggest that at least one antioxidant system may be involved in the local exercise-induced oxidative stress in these patients.

\section{Inflammatory markers after local muscle exercise}

Plasma inflammatory cytokine levels were unchanged by the exercise protocol and remained relatively constant throughout the postexercise period in both groups, suggesting that exercise did not induce systemic inflammation in parallel with the oxidative stress. Few studies have reported on inflammatory markers in response to exercise in COPD patients. The only available data on this topic, presented in a recent study, showed an increase in plasma TNF- $\alpha$ but not IL-6 level after $11 \mathrm{~min}$ of moderate-intensity exercise (constant-work-rate cycle ergometry) in severe COPD patients [30]. Plasma TNF- $\alpha$ levels showed a trend towards returning to resting values $20 \mathrm{~min}$ after exercise. This was not seen in the control group. The relationships between exercise, circulating cytokine levels and inflammation are not well understood and many contradictory results have been reported [34]. The type of physical activity and its intensity and duration are likely to be important factors in the discrepancies in these results [35]. The absence of exerciseinduced systemic inflammation in both of the present groups may therefore indicate that the exercise applied, localised to one muscle group for only a few minutes, was not sufficient to induce a systemic inflammatory process in healthy subjects or COPD patients.

The absence of exercise-induced inflammation in the present COPD group, despite local exercise-induced oxidative stress, suggests that mechanisms other than inflammation, such as alterations in the mitochondrial respiratory chain, xanthine oxidase activity or hypoxia, may promote this stress in COPD. Nevertheless, the marked systemic inflammation observed at rest may have acted indirectly on the magnitude of oxidative stress in response to exercise by altering the basal oxidant/antioxidant balance, as illustrated in figure 5 [36]. The 


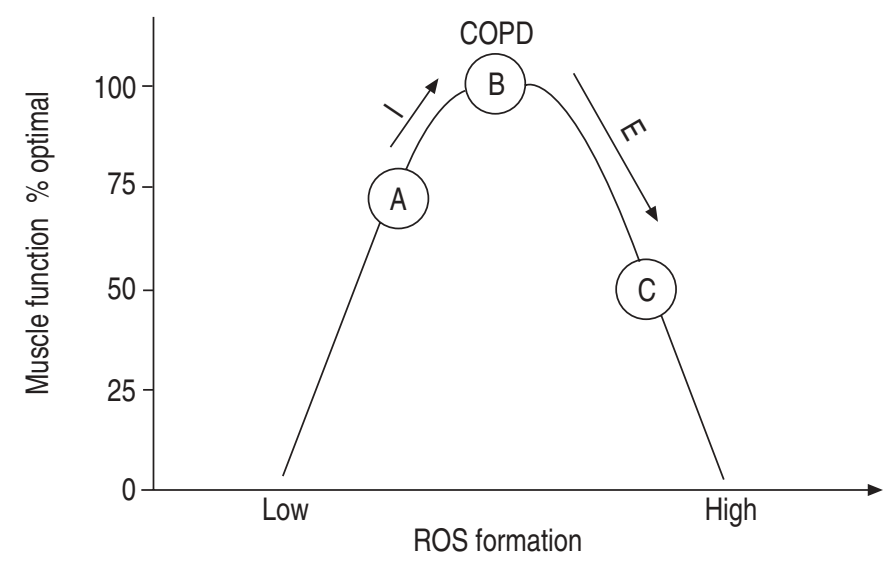

Fig. 5.-A proposed model for the relationship between basal inflammation, reactive oxygen species (ROS) formation and muscle function in chronic obstructive pulmonary disease (COPD) patients. A: basal state of healthy subjects; B: basal state of COPD patients with an increase in ROS exposure due to basal inflammation (I); C: decrease in muscle function following excessive ROS exposure in COPD patients due to exercise (E). In healthy subjects, exercise drives the basal state to point B but does not alter muscle function. Modified from [36].

marked basal systemic inflammation in COPD patients drives the basal state of oxidant production rightwards along the curve, near point B. Given this condition, a stimulus such as exercise of even light intensity, with a physiological increase in free radicals, is able to act as a trigger of oxidative stress in these patients, thereby inducing muscle alteration and fatigue, as seen at point $\mathrm{C}$.

The present results suggest that inflammatory and oxidant abnormalities coexist in chronic obstructive pulmonary disease at rest. They further suggest the possibility that these two processes interact in such a way as to trigger exercise-induced oxidative stress. The absence of local exercise-induced systemic inflammation, however, indicates that inflammation is unlikely to be the main cause of exercise-induced oxidative stress in chronic obstructive pulmonary disease, and that additional mechanisms, such as alterations in the mitochondrial respiratory chain or xanthine oxidase activity, may promote this stress in chronic obstructive pulmonary disease. Further studies on antioxidant supplementation or antiinflammatory drugs in chronic obstructive pulmonary disease patients would determine: 1) the exact interactions between oxidative stress and inflammation derangements suggested by the present study; and 2) whether or not these phenomena are involved in the myopathy of chronic obstructive pulmonary disease patients.

\section{References}

1. Repine J, Bast A, Lankhorst I. Oxidative stress in chronic obstructive pulmonary disease. Am J Respir Crit Care Med 1997; 156: 341-357.

2. McNee W. Oxidants/antioxidants and COPD. Chest 2000; 117: 5 Suppl. 1, S303-S317.

3. Couillard A, Koechlin C, Cristol JP, Varray A, Préfaut C. Evidence of local exercise-induced systemic oxidative stress in chronic obstructive pulmonary disease patients. Eur Respir J 2002; 20: 1123-1129.

4. Davies KJ, Quintanilha AT, Brooks GA, Packer L. Free radicals and tissue damage produced by exercise. Biochem Biophys Res Commum 1982; 107: 1198-1205.

5. Engelen MPKJ, Schols AMWJ, Does JD, Deutz NEP, Wouters EFM. Altered glutamate metabolism is associated with reduced muscle glutathione levels in patients with emphysema. Am J Respir Crit Care Med 2000; 161: 98-103.

6. American Thoracic Society, European Respiratory Society. Skeletal muscle dysfunction in chronic obstructive pulmonary disease. Am J Respir Crit Care Med 1999; 159: $\mathrm{S} 1-\mathrm{S} 40$.

7. Vina J, Servera E, Asensi M, et al. Exercise causes blood glutathione oxidation in chronic obstructive pulmonary disease: prevention by $\mathrm{O}_{2}$ therapy. J Appl Physiol 1996; 81: 2199-2202.

8. Heunks L, Vina $\mathbf{J}$, Cees $\mathbf{L}$, et al. Xanthine oxidase is involved in exercise-induced oxidative stress in chronic obstructive pulmonary disease. Am J Physiol 1999; 277: R1697-R1704.

9. Couillard A, Maltais F, Saey D, et al. Exercise-induced quadriceps oxidative stress and peripheral muscle dysfunction in patients with chronic obstructive pulmonary disease. Am J Respir Crit Care Med 2003; 167: 1664-1669.

10. Schols A, Buurman W, Staal-Van der Brekel A, Dentener M, Wouters F. Evidence for a relation between metabolic derangements and increased levels of inflammatory mediators in a subgroup of patients with chronic obstructive pulmonary disease. Thorax 1996; 51: 819-824.

11. Di Francia M, Barbier D, Mege JL, Orehek J. Tumor necrosis factor- $\alpha$ levels and weight loss in chronic obstructive pulmonary disease. Am J Respir Crit Care Med 1994; 150: $1453-1455$.

12. Agustí AGN, Noguera A, Sauleda J, Sala E, Pons J, Busquets X. Systemic effects of chronic obstructive pulmonary disease. Eur Respir $J$ 2003; 21: 347-360.

13. Reid MB, Li YP. Cytokines and oxidative signalling in skeletal muscle. Acta Physiol Scand 2001; 171: 225-232.

14. McNee W, Rahman I. Oxidants and antioxidants as therapeutic targets in chronic obstructive pulmonary disease. Am J Respir Crit Care Med 1999; 160: S58-S65.

15. Agustí AGN. Systemic effects of chronic obstructive pulmonary disease. In: Chadwick D, Goode JA, eds. Chronic Obstructive Pulmonary Disease: Pathogenesis to Treatment. Chichester, John Wiley \& Sons Ltd, 2001; pp. 242-254.

16. Noguera A, Batle S, Miralles C, et al. Enhanced neutrophil response in chronic obstructive pulmonary disease. Thorax 2001; 56: 432-437.

17. Pauwels RA, Buist AS, Calverley PM, Jenkins CR, Hurd SS. Global strategy for the diagnosis, management, and prevention of chronic obstructive pulmonary disease. NHLBI/ WHO Global Initiative for Chronic Obstructive Lung Disease (GOLD) Workshop summary. Am J Respir Crit Care Med 2001; 163: 1256-1276.

18. Quanjer PH, Tammeling GJ, Cote JE. Lung volumes and forced ventilatory flows. Eur Respir J 1993; 6: Suppl. 16, 540.

19. Voorips LE, Ravelli AC, Dongelmans P, Van Staveren W. A physical activity questionnaire for the elderly. Med Sci Sports Exerc 1991; 23: 974-979.

20. Yagi K. A simple fluorometric assay for lipoperoxide in blood plasma. Biochem Med 1976; 15: 212-216.

21. Witko-Sarsat V, Friedlander M, Khoa TN, et al. Advanced oxidation protein products as novels mediators of inflammation and monocyte activation in chronic renal failure. J Immunol 1998; 161: 2524-2532.

22. Vachier I, Chanez P, Le Doucen C, Damon M, Descomps B, Godard P. Enhancement of reactive oxygen species formation in stable and unstable asthmatic patients. Eur Respir $J$ 1994; 7: 1585-1592.

23. Cachia O, Leger C, Boulot $\mathrm{P}$, et al. Red blood cell vitamin $\mathrm{E}$ concentrations in fetuses are related to but lower than those in mothers during gestation. A possible association with maternal lipoprotein (a) plasma levels. Am J Obstet Gynecol 1995; 173: 42-51.

24. Andersen P, Adams RP, Sjogaard G, Thorboe A, Saltin B. Dynamic knee extension as model for study of isolated exercising muscle in humans. J Appl Physiol 1985; 59: 16471653. 
25. Serres I, Varray A, Vallet G, Micallef JP, Prefaut C. Improved skeletal muscle performance after individualised exercise training in patients with chronic obstructive pulmonary disease. J Cardiopulm Rehabil 1997; 4: 232-238.

26. Nielsen F, Mikkelsen BB, Nielsen BJ, Andersen HR, Grandjean P. Plasma malondialdehyde as biomarker for oxidative stress: reference interval and effects of life-style factors. Clin Chem 1997; 43: 1209-1214.

27. Han D, Loukianoff S, McLaughlin L. Oxidative stress indices: analytical aspects and significance. In: Sen CK, Packer L, Hanninen O, eds. Handbook of Oxidants and Antioxidants in Exercise. Amsterdam, Elsevier, 2000; pp. 433-483.

28. Kaneda H, Taguchi J, Ogasawara K, Aizawa T, Ohno M. Increased level of advanced oxidation protein products in patients with coronary artery disease. Atherosclerosis 2002; 162: 221-225.

29. Morena M, Cristol JP, Bosc JY, et al. Convective and diffusive losses of vitamin $\mathrm{C}$ during haemodiafiltration session: a contributive factor to oxidative stress in haemodialysis patients. Nephrol Dial Transplant 2002; 17: 422-427.

30. Rabinovich RA, Figuras M, Ardite E, et al. Increased tumour necrosis factor- $\alpha$ plasma levels during moderateintensity exercise in COPD patients. Eur Respir J 2003; 21: 789-794.

31. Rahman I, Morrison D, Donaldson K, McNee W. Systemic oxidative stress in asthma, COPD, and smokers. Am J Respir Crit Care Med 1996; 154: 1055-1066.

32. Draper HH, McGirr LG, Hadley M. The metabolism of malondialdehyde. Lipids 1986; 21: 305-307.

33. Rabinovich RA, Ardite E, Troosters T, et al. Reduced muscle redox capacity after endurance training in patients with chronic obstructive pulmonary disease. Am J Respir Crit Care Med 2001; 164: 1114-1118.

34. Northoff H, Weinstock C, Berg A. The cytokine response to strenuous exercise. Int J Sport Med 1994; 15: Suppl. 3, S167S171.

35. Pedersen BK, Ostrowski K, Rohde T, Bruunsgaard H. The cytokine response to strenuous exercise. Can J Physiol Pharmacol 1998; 76: 505-511.

36. Reid MB. Muscle fatigue: mechanisms and regulation. In: Sen CK, Packer L, Hanninen O, eds. Handbook of Oxidants and Antioxidants in Exercise. Amsterdam, Elsevier, 2000; pp. 599-629. 